INTERNATIONAL JOURNAL FOR NUMERICAL METHODS IN ENGINEERING

Int. J. Numer. Meth. Engng 2000; 00:1-6 Prepared using nmeauth.cls [Version: 2002/09/18 v2.02]

\title{
Linearization techniques for band structure calculations in absorbing photonic crystals
}

\author{
C. Effenberger ${ }^{1 \dagger *}$, D. Kressner ${ }^{1 \dagger}$ and C. Engström ${ }^{1,2 \ddagger}$ \\ ${ }^{1}$ Seminar for Applied Mathematics, ETH Zurich, Rämistrasse 101, 8092 Zurich, Switzerland \\ ${ }^{2}$ Electromagnetic Fields and Microwave Electronics Laboratory, \\ ETH Zurich, Gloriastrasse 35, 8092 Zurich, Switzerland
}

\begin{abstract}
SUMMARY
Band structure calculations for photonic crystals require the numerical solution of eigenvalue problems. In this paper, we consider crystals composed of lossy materials with frequency-dependent permittivities. Often, these frequency dependencies are modeled by rational functions, such as the Lorentz model, in which case the eigenvalue problems are rational in the eigenvalue parameter. After spatial discretization using an interior penalty discontinuous Galerkin method, we employ a recently developed linearization technique to deal with the resulting rational matrix eigenvalue problems. In particular, the efficient implementation of Krylov subspace methods for solving the linearized eigenvalue problems is investigated in detail. Numerical experiments demonstrate that our new approach is considerably cheaper in terms of memory and computing time requirements compared to the naive approach of turning the rational eigenvalue problem into a polynomial eigenvalue problem and applying standard linearization techniques. Copyright (c) 2000 John Wiley \& Sons, Ltd.
\end{abstract}

\section{Introduction}

This paper is concerned with wave propagation in periodic absorbing media with frequencydependent material properties. In particular, we consider electromagnetic wave propagation in two-dimensional photonic crystals. In lossless materials, the propagating waves are determined by the spectrum of an underlying PDE eigenvalue problem. Numerical methods for computing this spectrum are well established for frequency-independent materials [10, 3, 7, 29]. Only rather recently, complex dispersion relations $\omega(\vec{k})$ in absorbing media with frequencydependent material properties have been investigated numerically $[27,24,15,22,9]$. The frequency-dependent case gives rise to nonlinear eigenvalue problems, for which the development of robust numerical methods is a subtle issue.

\footnotetext{
*Correspondence to: C. Effenberger, Seminar for Applied Mathematics, ETH Zurich; Rämistrasse 101, 8092 Zurich, Switzerland; E-mail: cedric.effenberger@sam.math.ethz.ch

${ }^{\dagger}$ The work of C. Effenberger and D. Kressner has been supported by the SNF research module Robust numerical methods for solving nonlinear eigenvalue problems within the SNF ProDoc Efficient Numerical Methods for Partial Differential Equations.

${ }^{\ddagger}$ The work of C. Engström has been supported by a Marie Curie Intra-European Fellowship of the European Community; contract/grant number: PIEF-GA-2009-237397.
} 
In our setting, we assume that the waves propagate in a nonmagnetic material with permittivity $\epsilon$ depending on the first two spatial coordinates $x_{1}, x_{2}$ as well as on the frequency $\omega$, but not depending on the third coordinate $x_{3}$. As usual, the electromagnetic wave $(E, H)$ is decomposed into transverse electric (TE) polarized waves $\left(E_{1}, E_{2}, 0,0,0, H_{3}\right)$ and transverse magnetic (TM) polarized waves $\left(0,0, E_{3}, H_{1}, H_{2}, 0\right)$ [17]. This decomposition reduces the full $3 D$ Maxwell equations to scalar $2 D$ Helmholtz equations in $H_{3}$ and $E_{3}$, respectively. For simplicity, the discussion will focus on TM polarized waves, but the developed numerical methods can be applied to the TE case as well. By a Bloch ansatz, the original problem on $\mathbb{R}^{2}$ is turned into a family of eigenvalue problems in $\omega$ posed on the unit cell.

For the finite element discretization of these eigenvalue problems, we use a discontinuous Galerkin framework with curved high-order finite elements. This leads to finite-dimensional eigenvalue problems, which are nonlinear in the eigenvalue parameter $\omega$ due to the frequencydependency of $\epsilon$. The nature of this nonlinearity depends on how the material properties are modeled. Solid materials are typically represented by a sum of Lorentz terms [17], which leads to rational nonlinearities.

Compared to linear eigenvalue problems, the robust numerical solution of nonlinear eigenvalue problems is much more difficult. Newton-type methods as proposed in $[4,30,31,19]$ have excellent local convergence properties but bear the danger that eigenvalues in a specified region of interest could be missed. In special cases, this lack of robustness can be addressed by employing a minmax characterization [35, 36, 37]. Unfortunately, such a characterization does not exist for lossy materials. In the case of rational eigenvalue problems, the most straightforward approach is to multiply by the common denominator and apply standard linearization techniques to the resulting polynomial eigenvalue problem $[12,23]$. However, with an increasing number of poles, the degree of the intermediate polynomial eigenvalue problem quickly becomes large, leading to a severe magnification of the problem size during the linearization process. In this paper, an alternative linearization based directly on the rational formulation is proposed along the lines of recent work by Bai and Su [32]. This approach leads to significantly smaller linearizations, especially when the coefficient matrices associated with the rational terms are of low rank. Combined with structure-exploiting Krylov subspace techniques, we demonstrate that this linearization leads to an efficient and robust numerical method for band structure calculations.

The rest of this paper is organized as follows. In Section 2, we describe the mathematical setting of complex band structure calculation and its spatial discretization by an interior penalty discontinuous Galerkin method. Section 3 discusses two different linearization techniques for addressing the resulting rational matrix eigenvalue problem. As the linearized eigenvalue problem is solved by an inverse Arnoldi method, Section 4 is devoted to the efficient numerical inversion for our newly proposed linearization. Finally, Section 5 contains a number of numerical experiments, which demonstrate that the approach proposed in this paper leads to improved performance compared to the standard approach.

\section{Band structures of photonic crystals}

Non-magnetic two-dimensional photonic crystals are characterized by a periodic frequencydependent permittivity $\epsilon$. By scaling the lattice constants to unit length, the lattice $\Gamma$ becomes $\mathbb{Z}^{2}$ with the unit cell $\Omega=(0,1]^{2}$. In the TM case, the third coordinate $E_{3}$ of the electric field 


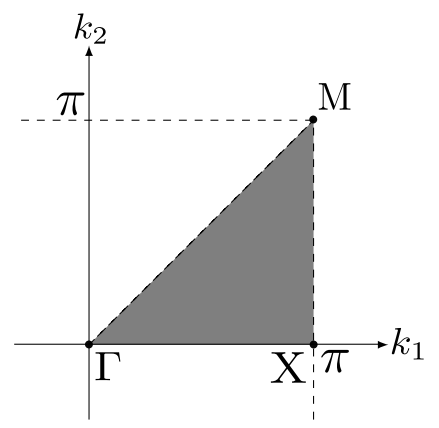

Figure 1. The irreducible Brillouin zone with the symmetry points $\Gamma, X$, and $M$.

satisfies the equation

$$
-\Delta E_{3}(\vec{x})=\frac{\omega^{2}}{\mathrm{c}^{2}} \epsilon(\vec{x}, \omega) E_{3}(\vec{x}), \quad \vec{x}=\left(x_{1}, x_{2}\right),
$$

where $\omega$ is the time frequency and $c$ is the speed of light in vacuum. The main tool to study spectral properties of PDEs with periodic coefficients is Floquet-Bloch theory [25, 26, 11, 20]. A Bloch solution to (1) is a nonzero function of the form

$$
E_{3}(\vec{x})=\mathrm{e}^{\mathrm{i} \vec{k} \cdot \vec{x}} u(\vec{x}),
$$

where $u$ is a periodic function on $\Gamma$ and $\vec{k} \in \Omega^{*}=(-\pi, \pi]^{2}$ denotes the wave vector. By inserting the Bloch ansatz (2), the original problem (1) posed on the infinite domain $\mathbb{R}^{2}$ becomes

$$
-(\nabla+\mathrm{i} \vec{k}) \cdot(\nabla+\mathrm{i} \vec{k}) u=\frac{\omega^{2}}{\mathrm{c}^{2}} \epsilon(\vec{x}, \omega) u,
$$

which is a family of eigenvalue problems in $\omega$ on the unit cell $\Omega$ with periodic boundary conditions. When calculating the dispersion relations $\omega(\vec{k})$ numerically, frequently only a selection of wave vectors $\vec{k}$ along the line segments between the points $\Gamma, X$, and $M$, as shown in Figure 1, is considered. The triangular path formed by these points is called the boundary of the irreducible Brillouin zone [18].

We assume that electromagnetic energy may be transferred into the material, but energy is not transferred from the material into the electromagnetic field. In other words, the material is assumed to be passive [5, 17], which corresponds to the condition

$$
\omega \epsilon(\omega) \in \mathbb{C}_{+}=\{z \in \mathbb{C}: 0 \leq \arg z<\pi, z \neq 0\} \quad \forall \omega \in \mathbb{C}_{+} .
$$

An important consequence of $(4)$ is $\Im \epsilon(\omega) \geq 0$ for $\omega>0$. The spectral problem (3) with a passive material model was analyzed in $[8]$, where it was proved that the spectrum is discrete. In principle, any general space-dependent permittivity can be handled when the eigenvalue problem is discretized with finite elements. However, for simplicity, we restrict the permittivity to be piecewise constant with respect to a finite domain partitioning $\Omega=\Omega_{1} \cup \cdots \cup \Omega_{S}$ :

$$
\epsilon(\vec{x}, \omega)=\sum_{s=1}^{S} \epsilon_{s}(\omega) I_{s}(\vec{x})
$$


where $I_{s}$ is the indicator function for the subdomain $\Omega_{s}$. In general, the permittivities $\epsilon_{s}$ are complicated unknown functions in the frequency $\omega$. It is therefore common to model this frequency dependency by fitting a rational function satisfying (4) to measurement data. The resulting Lorentz model takes the form

$$
\epsilon_{s}(\omega)=\alpha_{s}+\sum_{\ell=1}^{L_{s}} \frac{\xi_{s, \ell}^{2}}{\eta_{s, \ell}^{2}-\omega^{2}-\mathrm{i} \gamma_{s, \ell} \omega} .
$$

\subsection{Discontinuous Galerkin approximation}

In the following, we discretize a weak formulation of the PDE eigenvalue problem (3) with a discontinuous finite element method $[2,1,14]$. The $p$-version of the finite element method uses polynomial basis functions on a fixed (coarse) mesh and achieves convergence by enhancement of the polynomial degree. More specifically, we use the $p$-version of the symmetric interior penalty method (SIP) in this paper since the discretization with a high-order method results, for a given accuracy, in comparably small matrices. Moreover, discontinuous Galerkin methods, such as SIP, are more flexible in the choice of basis functions and the mesh design.

Let $\mathcal{V}_{h}^{p}$ denote the space of piecewise polynomials of degree $p$ on a triangulation $\mathcal{T}_{h}$ of the unit cell $\Omega=\cup_{K \in \mathcal{T}_{h}} K$. Consider two triangles $K_{+}, K_{-} \in \mathcal{T}$ with the outward pointing normals $\vec{n}_{+}, \vec{n}_{-}$on the shared edge $\partial K_{+} \cap \partial K_{-}$. The averages $\{\cdot\}$ and jumps [·] of a piecewise smooth function $w$ on $K_{+} \cup K_{-}$are then defined as usual:

$$
\begin{aligned}
\{w\} & =\frac{1}{2}\left(w_{+}+w_{-}\right), & {[w] } & =w_{+} \vec{n}_{+}+w_{-} \vec{n}_{-}, \\
\{\nabla w\} & =\frac{1}{2}\left(\nabla w_{+}+\nabla w_{-}\right), & {[\nabla w] } & =\frac{\partial w_{+}}{\partial \vec{n}_{+}}+\frac{\partial w_{-}}{\partial \vec{n}_{-}} .
\end{aligned}
$$

For $u_{h}, v_{h} \in \mathcal{V}_{h}^{p}$ we consider the sesquilinear forms

$$
\begin{aligned}
a_{\vec{k}}\left(u_{h}, v_{h}\right) & =\int_{\Omega} \nabla u_{h} \cdot \nabla \bar{v}_{h} \mathrm{~d} \vec{x}-\int_{\mathcal{E}}\left\{\nabla u_{h}\right\} \cdot\left[\bar{v}_{h}\right] \mathrm{d} \vec{x}-\int_{\mathcal{E}}\left\{\nabla v_{h}\right\} \cdot\left[\bar{u}_{h}\right] \mathrm{d} \vec{x} \\
& +\int_{\mathcal{E}} \beta\left[u_{h}\right] \cdot\left[\bar{v}_{h}\right] \mathrm{d} \vec{x}-2 \mathrm{i} \int_{\Omega} \vec{k} \cdot \nabla u_{h} \bar{v}_{h} \mathrm{~d} \vec{x}+\int_{\Omega} \vec{k} \cdot \vec{k} u_{h} \bar{v}_{h} \mathrm{~d} \vec{x},
\end{aligned}
$$

with $\mathcal{E}$ denoting the set of all edges in $\mathcal{T}_{h}$, and

$$
b_{\omega}\left(u_{h}, v_{h}\right)=\int_{\Omega} \epsilon(\vec{x}, \omega) u_{h} \bar{v}_{h} \mathrm{~d} \vec{x} .
$$

Note that the periodic boundary conditions are imposed weakly by identifying opposite sides of the unit cell and enforcing periodicity of the solution via the corresponding penalty terms in $(9)$; see $[34,9]$.

The discretized nonlinear eigenvalue problem can now be stated as follows: Find $\omega \in \mathbb{C}$ and a nonzero $u_{h} \in \mathcal{V}_{h}^{p}$ such that

$$
a_{\vec{k}}\left(u_{h}, v_{h}\right)=\omega^{2} b_{\omega}\left(u_{h}, v_{h}\right)
$$

holds for all $v_{h} \in \mathcal{V}_{h}^{p}$. A numerical method based on (11) is symmetric and stable provided that the penalty parameter $\beta$ is sufficiently large $[1,34]$. Based on the experiments from [9], we use

$$
\beta=20 \frac{(p+1)(p+2)}{2} \frac{|\partial K|}{|K|},
$$


where $|\partial K|$ denotes the length of the edge and $|K|$ designates the area of the smaller of the adjacent triangles. The global approximation $u$ is computed as a direct sum of local solutions $u^{K}$ at every triangle $K$ in the mesh.

To generate the mesh, we use the software package $E m c^{2}$ [28]. Frequently, parts of the geometry are curved, for example, a circular interface between an inclusion and the matrix. In order to obtain high accuracy by increasing the polynomial degree $p$ of the finite elements, it is therefore mandatory to use curved triangles combined with a Gordon-Hall blending $[16,14]$. To assemble (11), we build on the MATLAB version [14] of NUDG++ (www.nudg.org). The local matrices on a triangle $K$ are assembled into global matrices, and finally, the discretized nonlinear eigenvalue problem takes the form

$$
G u-\omega^{2} \sum_{s=1}^{S} \epsilon_{s}(\omega) M_{s} u=0,
$$

where $u$ is now a vector containing the coefficients of $u_{h}$. The number of degrees of freedom $(N)$ is $\frac{1}{2}(p+1)(p+2)$ times the number of triangles in the mesh. The finite element discretization error for this formulation was addressed in [9]. The focus of this paper is on efficient algorithms for solving the discretized problem (13).

\section{Linearization of rational eigenvalue problems}

In this section, we discuss the numerical solution of the finite-dimensional nonlinear eigenvalue problem (13). For the moment, we consider a two-component model $(S=2)$ with one of the materials having constant permittivity $\alpha_{1}$. For the other material, the permittivity is described by the Lorentz model (6), resulting in the rational eigenvalue problem

$$
G u-\omega^{2} \alpha_{1} M_{1} u-\omega^{2}\left(\alpha_{2}+\sum_{\ell=1}^{L} \frac{\xi_{\ell}^{2}}{\eta_{\ell}^{2}-\omega^{2}-\mathrm{i} \gamma_{\ell} \omega}\right) M_{2} u=0 .
$$

Later on, in Section 3.3, we will comment on the extension to arbitrarily many components.

\subsection{Linearization via a polynomial eigenvalue problem}

Simply through multiplication by the denominators, the rational eigenvalue problem (14) can be turned into a polynomial eigenvalue problem. More specifically, we obtain

$$
\left[\prod_{\ell=1}^{L}\left(\eta_{\ell}^{2}-\omega^{2}-\mathrm{i} \gamma_{\ell} \omega\right)\right]\left(G-\omega^{2}\left(\alpha_{1} M_{1} u+\alpha_{2} M_{2}\right)\right) u-\omega^{2}\left[\sum_{\ell=1}^{L} \xi_{\ell}^{2} \prod_{m \neq \ell}\left(\eta_{m}^{2}-\omega^{2}-\mathrm{i} \gamma_{m} \omega\right)\right] M_{2} u=0 .
$$

The eigenvalues of (15) coincide with the eigenvalues of (14), provided that $\omega$ is bounded away from the poles. Sorting coefficients, the matrix polynomial (15) can be written in the form

$$
\omega^{2 L+2} P_{2 L+2} u+\omega^{2 L+1} P_{2 L+1} u+\cdots+P_{0} u=0,
$$

for certain matrices $P_{0}, \ldots, P_{2 L+2}$. The usual way to solve (16) is to apply a standard eigenvalue solver to an equivalent linear eigenvalue problem

$$
\mathcal{L}_{1} u=\omega \mathcal{L}_{2} u .
$$


This linearization is not unique [23]; a popular choice are companion-type linearizations, one variant of which gives

$$
\mathcal{L}_{1}=\left[\begin{array}{cccc}
P_{0} & & & \\
& I & & \\
& & \ddots & \\
& & & I
\end{array}\right], \quad \mathcal{L}_{2}=\left[\begin{array}{cccc}
-P_{1} & \cdots & -P_{2 L+1} & -P_{2 L+2} \\
I & & & \\
& \ddots & &
\end{array}\right] .
$$

An obvious disadvantage of this approach is the increase of the dimension from $N$ to $(2 L+2) \cdot N$. More subtle disadvantages include a certain loss of sparsity and numerical instability due to numerical cancellation during the process of forming $P_{j}$.

\subsection{Direct linearization}

To avoid the above-mentioned disadvantages, we consider a direct linearization of (14) without the intermediate transformation to a matrix polynomial, based on the approach proposed by Bai and $\mathrm{Su}$ [32]. To apply this approach, we first bring the rational terms in (14) to proper form:

$$
\frac{\xi_{\ell}^{2} \omega^{2}}{\eta_{\ell}^{2}-\omega^{2}-\mathrm{i} \gamma_{\ell} \omega}=-\xi_{\ell}^{2}+\frac{\xi_{\ell}^{2}\left(\eta_{\ell}^{2}-\mathrm{i} \gamma_{\ell} \omega\right)}{\eta_{\ell}^{2}-\omega^{2}-\mathrm{i} \gamma_{\ell} \omega}
$$

The second term in this sum can be represented as $b_{\ell}^{T}\left(A_{\ell}-\omega E_{\ell}\right)^{-1} b_{\ell}$, where

$$
A_{\ell}=\frac{2 \theta_{\ell}}{\xi_{\ell}^{2}}\left[\begin{array}{cc}
\theta_{\ell}-\frac{1}{2} \mathrm{i} \gamma_{\ell} & 0 \\
0 & \theta_{\ell}+\frac{1}{2} \mathrm{i} \gamma_{\ell}
\end{array}\right], \quad E_{\ell}=\frac{2 \theta_{\ell}}{\xi_{\ell}^{2}}\left[\begin{array}{cc}
1 & 0 \\
0 & -1
\end{array}\right], \quad b_{\ell}=\left[\begin{array}{c}
\theta_{\ell}-\frac{1}{2} \mathrm{i} \gamma_{\ell} \\
\theta_{\ell}+\frac{1}{2} \mathrm{i} \gamma_{\ell}
\end{array}\right],
$$

with $\theta_{\ell}=\sqrt{\eta_{\ell}^{2}-\frac{1}{4} \gamma_{\ell}^{2}}$. Setting $A=\operatorname{diag}\left(A_{1}, \ldots, A_{L}\right), E=\operatorname{diag}\left(E_{1}, \ldots, E_{L}\right)$, and $b=$ $\left[b_{1}^{T}, \ldots, b_{L}^{T}\right]^{T}$, the eigenvalue problem (14) becomes

$$
\left(\hat{G}-\omega^{2} \hat{M}-b^{T}(A-\omega E)^{-1} b M_{2}\right) u=0,
$$

where

$$
\hat{G}=G+\sum_{\ell=1}^{L} \xi_{\ell}^{2} M_{2}, \quad \hat{M}=\alpha_{1} M_{1}+\alpha_{2} M_{2} .
$$

To proceed from (20), we note that $M_{2}$ is the mass matrix for the FE basis functions supported on the frequency-dependent subdomain $\Omega_{2}$. By a suitable ordering of the nodes, $M_{2}$ takes the form $M_{2}=\operatorname{diag}\left(0, \check{M}_{2}\right)$ with a symmetric positive definite matrix $\check{M}_{2} \in \mathbb{R}^{N_{2} \times N_{2}}$. Note that $N_{2}<N$ corresponds to the number of basis functions supported on $\Omega_{2}$. Performing a Cholesky factorization of $\check{M}_{2}$, we can therefore write $M_{2}=F^{T} F$ for some $F \in \mathbb{R}^{N_{2} \times N}$. This gives

$$
\begin{aligned}
b^{T}(A-\omega E)^{-1} b M_{2} & =b^{T}(A-\omega E)^{-1} b \otimes F^{T} F \\
& =\mathcal{B}^{T}(\mathcal{A}-\omega \mathcal{E})^{-1} \mathcal{B}
\end{aligned}
$$

where $\mathcal{B}:=b \otimes F, \mathcal{A}:=A \otimes I_{N_{2}}, \mathcal{E}:=E \otimes I_{N_{2}}$. Note that $\otimes$ denotes the Kronecker product of two matrices; see [13] for details.

Combined with (20),

$$
\left(\hat{G}-\omega^{2} \hat{M}-\mathcal{B}^{T}(\mathcal{A}-\omega \mathcal{E})^{-1} \mathcal{B}\right) u=0,
$$


the eigenvalue problem can now be linearized by introducing the auxiliary vectors $v=$ $-(\mathcal{A}-\omega \mathcal{E})^{-1} \mathcal{B} u$ and $w=\omega u$ :

$$
\left[\begin{array}{ccc}
\hat{G} & \mathcal{B}^{T} & 0 \\
\mathcal{B} & \mathcal{A} & 0 \\
0 & 0 & I
\end{array}\right]\left[\begin{array}{c}
u \\
v \\
w
\end{array}\right]=\omega\left[\begin{array}{ccc}
0 & 0 & \hat{M} \\
0 & \mathcal{E} & 0 \\
I & 0 & 0
\end{array}\right]\left[\begin{array}{c}
u \\
v \\
w
\end{array}\right] .
$$

This is a generalized linear eigenvalue problem of order $2 N+2 L N_{2}$, which compares favorably with the order $2 N+2 L N$ of the linearized polynomial eigenvalue problem.

\subsection{Extension to $S>2$ materials}

The derivation above can be directly extended to $S$ subdomains $\Omega_{s}, s=1, \ldots, S$, for which the frequency dependency is described by the parameters $\alpha_{s}, \xi_{s, \ell}, \eta_{s, \ell}, \gamma_{s, \ell}$ in the Lorentz model (6). Based on these parameters, matrices $A_{s, \ell}, E_{s, \ell}$, and vectors $b_{s, \ell}, s=1, \ldots, S, \ell=1, \ldots, L_{s}$, are defined analogously as in (19). After factorizing the mass matrices $M_{s}=F_{s}^{T} F_{s}, F_{s} \in \mathbb{R}^{N_{s} \times N}$ as before and defining

$$
\begin{aligned}
\hat{G} & =G+\sum_{s=1}^{S} \sum_{\ell=1}^{L_{s}} \xi_{s, \ell}^{2} M_{s}, \\
\hat{M} & =\alpha_{1} M_{1}+\cdots+\alpha_{S} M_{S}, \\
\mathcal{A} & =\operatorname{diag}\left(A_{1} \otimes I_{N_{1}}, \ldots, A_{S} \otimes I_{N_{S}}\right), \\
\mathcal{E} & =\operatorname{diag}\left(E_{1} \otimes I_{N_{1}}, \ldots, E_{S} \otimes I_{N_{S}}\right), \\
\mathcal{B} & =\left[\left(b_{1} \otimes F_{1}\right)^{T}, \ldots,\left(b_{S} \otimes F_{S}\right)^{T}\right]^{T},
\end{aligned}
$$

where

$$
\begin{aligned}
A_{s} & =\operatorname{diag}\left(A_{s, 1}, \ldots, A_{s, L_{s}}\right), \\
E_{s} & =\operatorname{diag}\left(E_{s, 1}, \ldots, E_{s, L_{s}}\right), \\
b_{s} & =\left[b_{s, 1}^{T}, \ldots, b_{s, L_{s}}^{T}\right]^{T},
\end{aligned}
$$

for $s=1, \ldots, S$, we again obtain a linearized eigenvalue problem of precisely the form (22). The total size of the linearization is only $2 N+2 L_{1} N_{1}+\cdots+2 L_{S} N_{S}$ as opposed to $2 N+2 L_{1} N+\cdots+2 L_{S} N$ for the polynomial approach.

Up to this point, the derivation of the direct linearization has been a fairly straightforward application of the ideas proposed by Bai and $\mathrm{Su}$ [32]. However, to be able to use this linearization in an iterative eigenvalue solver, special care of the particular structure of our problem needs to be taken. This will be discussed in the following section.

\section{Solution of the linearized eigenvalue problem}

Both linearizations discussed in Section 3 lead to a linear eigenvalue problem of the form $\mathcal{L}_{1} u=\omega \mathcal{L}_{2} u$. The eigenvalues relevant to our application are located in a rectangle enclosing a part of the real axis ranging from 0 up to a certain threshold frequency. Our strategy for determining these eigenvalues is to first compute the eigenvalues of smallest magnitude and 
then exclude those lying outside of the rectangle. Premultiplying the eigenvalue problem by $\mathcal{L}_{1}^{-1}$ and dividing by $\omega$ yields

$$
\mathcal{L}_{1}^{-1} \mathcal{L}_{2} u=\omega^{-1} u \text {. }
$$

Consequently, $\omega$ is an eigenvalue for $\mathcal{L}_{1} u=\omega \mathcal{L}_{2} u$ if and only if $\omega^{-1}$ is an eigenvalue of the matrix $\Phi:=\mathcal{L}_{1}^{-1} \mathcal{L}_{2}$. Thus, our aim becomes the computation of eigenvalues of largest magnitude for $\Phi$, a problem which can be easily addressed using standard Krylov subspace methods, such as the implicitly restarted Arnoldi algorithm [21].

These Krylov subspace methods are based on repeated matrix-vector multiplication with $\Phi$, which, in particular, requires the efficient inversion of $\mathcal{L}_{1}$. For the companion linearization (18), this task is reduced to the inversion of the coefficient matrix $P_{0}$ thanks to the special structure of $\mathcal{L}_{1}$. It is fairly easy to see from (15) that $P_{0}$ is, in fact, a scalar multiple of the sparse matrix $G$. Hence, employing a sparse direct solver will be efficient. For the direct linearization (22), the question of how the structure in $\mathcal{L}_{1}$ can be exploited is much more subtle and the rest of this section will be devoted to this issue.

First, we recall that for the direct linearization (22), the reciprocal eigenvalue problem (23) takes the form

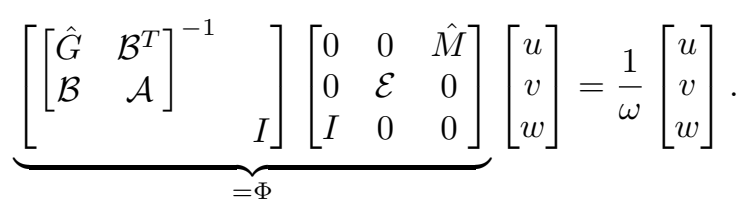

We then factorize

$$
\left[\begin{array}{cc}
\hat{G} & \mathcal{B}^{T} \\
\mathcal{B} & \mathcal{A}
\end{array}\right]=\left[\begin{array}{cc}
I & \mathcal{B}^{T} \mathcal{A}^{-1} \\
0 & I
\end{array}\right]\left[\begin{array}{cc}
\hat{G}-\mathcal{B}^{T} \mathcal{A}^{-1} \mathcal{B} & 0 \\
0 & \mathcal{A}
\end{array}\right]\left[\begin{array}{cc}
I & 0 \\
\mathcal{A}^{-1} \mathcal{B} & I
\end{array}\right]
$$

From the definitions of $\hat{G}, \mathcal{A}$, and $\mathcal{B}$ in Section 3.2, we find that

$$
\hat{G}-\mathcal{B}^{T} \mathcal{A}^{-1} \mathcal{B}=\hat{G}-b^{T} A^{-1} b \otimes F^{T} F=\hat{G}-\sum_{\ell=1}^{L} b_{\ell}^{T} A_{\ell}^{-1} b_{\ell} \otimes M_{2}=\hat{G}-\sum_{\ell=1}^{L} \xi_{\ell}^{2} M_{2}=G .
$$

Inserting this relation into (25) and inverting yields

$$
\left[\begin{array}{cc}
\hat{G} & \mathcal{B}^{T} \\
\mathcal{B} & \mathcal{A}
\end{array}\right]^{-1}=\left[\begin{array}{cc}
I & 0 \\
-\mathcal{A}^{-1} \mathcal{B} & I
\end{array}\right]\left[\begin{array}{cc}
G^{-1} & 0 \\
0 & \mathcal{A}^{-1}
\end{array}\right]\left[\begin{array}{cc}
I & -\mathcal{B}^{T} \mathcal{A}^{-1} \\
0 & I
\end{array}\right]=\left[\begin{array}{cc}
G^{-1} & -G^{-1} \mathcal{B}^{T} \mathcal{A}^{-1} \\
-\mathcal{A}^{-1} \mathcal{B} G^{-1} & \mathcal{A}^{-1}+\mathcal{A}^{-1} \mathcal{B} G^{-1} \mathcal{B}^{T} \mathcal{A}^{-1}
\end{array}\right] .
$$

Hence, the matrix-vector product $\left[\tilde{u}^{T}, \tilde{v}^{T}, \tilde{w}^{T}\right]^{T}:=\Phi\left[u^{T}, v^{T}, w^{T}\right]^{T}$ is given by the formulas

$$
\begin{aligned}
\tilde{u} & =G^{-1} \hat{M} w-G^{-1} \mathcal{B}^{T} \mathcal{A}^{-1} \mathcal{E} v=G^{-1}\left(\hat{M} w-\mathcal{B}^{T} \mathcal{A}^{-1} \mathcal{E} v\right) \\
\tilde{v} & =\mathcal{A}^{-1} \mathcal{E} v+\mathcal{A}^{-1} \mathcal{B} G^{-1} \mathcal{B}^{T} \mathcal{A}^{-1} \mathcal{E} v-\mathcal{A}^{-1} \mathcal{B} G^{-1} \hat{M} w=\mathcal{A}^{-1} \mathcal{E} v-\mathcal{A}^{-1} \mathcal{B} \tilde{u} \\
\tilde{w} & =u
\end{aligned}
$$

Consequently, the major computational work when applying $\Phi$ consists in solving a linear system with the stiffness matrix $G$. All the remaining operations are comparatively cheap:

- The matrix-vector product $\hat{M} w$ can be evaluated efficiently since $\hat{M}$ is sparse. 
- The matrix-vector product $\mathcal{B}^{T} \mathcal{A}^{-1} \mathcal{E} v$ can be rewritten as

$$
\mathcal{B}^{T} \mathcal{A}^{-1} \mathcal{E} v=\left(b^{T} A^{-1} E \otimes F^{T}\right) v=\left(\left[\begin{array}{c}
1 \\
-1 \\
\vdots \\
1 \\
-1
\end{array}\right] \otimes F^{T}\right) v=\left(\left[\begin{array}{c}
1 \\
\vdots \\
1
\end{array}\right] \otimes\left[\begin{array}{c}
1 \\
-1
\end{array}\right] \otimes F^{T}\right) v
$$

using the definitions of $\mathcal{B}, \mathcal{A}, \mathcal{E}$ in Section 3.2. By partitioning $v$ as $v=\left[v_{1}^{T}, \ldots, v_{2 L}^{T}\right]^{T}$, where $v_{1}, \ldots, v_{2 L}$ are vectors of dimension $N_{2}$ each, we obtain

$$
\mathcal{B}^{T} \mathcal{A}^{-1} \mathcal{E} v=F^{T}\left(v_{1}-v_{2}+\cdots-\cdots+v_{2 L-1}-v_{2 L}\right) .
$$

Thus, the main computational effort is the multiplication by $F^{T}$.

- The matrix-vector product $\mathcal{A}^{-1} \mathcal{E} v$ amounts to rescaling the entries of the vector $v$ because

$$
\mathcal{A}^{-1} \mathcal{E}=A^{-1} E \otimes I_{N_{2}}=\operatorname{diag}\left(A_{1}^{-1} E_{1}, \ldots, A_{L}^{-1} E_{L}\right) \otimes I_{N_{2}}
$$

is a diagonal matrix.

- The matrix-vector product $\mathcal{A}^{-1} \mathcal{B} \tilde{u}$ can be recast as

$$
\mathcal{A}^{-1} \mathcal{B} \tilde{u}=\left(A^{-1} b \otimes F\right) \tilde{u}=\left[\begin{array}{c}
\frac{\xi_{1}^{2}}{2 \theta_{1}} \\
\frac{\xi_{1}^{2}}{2 \theta_{1}} \\
\vdots \\
\frac{\xi_{L}^{2}}{2 \theta_{L}} \\
\frac{\xi_{L}^{2}}{2 \theta_{L}}
\end{array}\right] \otimes(F \tilde{u})=\left[\begin{array}{c}
\frac{\xi_{1}^{2}}{2 \theta_{1}} \\
\vdots \\
\frac{\xi_{L}^{2}}{2 \theta_{L}}
\end{array}\right] \otimes\left[\begin{array}{c}
1 \\
1
\end{array}\right] \otimes(F \tilde{u})
$$

Hence, the computational cost is essentially that of applying $F$ to $\tilde{u}$.

As the stiffness matrix $G$ is Hermitian and - for nonzero wave vectors $\vec{k}$ - also positive definite, we employ a sparse Cholesky decomposition for solving linear systems with $G$. The decomposition is performed in a preprocessing step before the Krylov subspace method is invoked. Unfortunately, $G$ depends on the wave vector; therefore, it needs to be refactorized whenever the wave vector changes. Still, we benefit significantly from reusing the Cholesky factors since the number of wave vectors under consideration is typically much smaller than the total number of linear systems to be solved.

\section{Numerical examples}

In order to evaluate the numerical method proposed in Section 4, we consider the following setup: A disk of radius $r=0.2$ consisting of a material with frequency-dependent permittivity is located in the center of the unit cell; see Figure 2. The rest of the unit cell is filled with air (relative permittivity $\epsilon_{1}=1$ ). The permittivity of the frequency-dependent material is governed by the Lorentz model (6), leading to a rational eigenvalue problem of the form (14).

We may solve this eigenvalue problem via the standard linearization based on an intermediate transformation to a polynomial eigenvalue problem (see Section 3.1) or via 


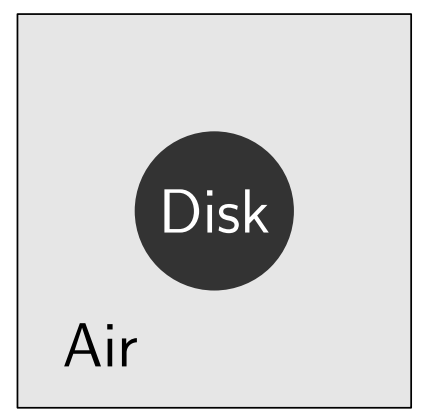

Figure 2. Unit cell of the benchmark setup

the direct linearization developed in Section 3.2. The computations have been performed under MATLAB 7.10 on a cluster of 16 AMD Opteron 8356 processors with 64 GB of shared memory. The MATLAB interface to UMFPACK [6] has been utilized to carry out sparse direct factorizations. The linearized eigenvalue problems have been solved by the MATLAB interface eigs to ARPACK [21]. All reported computing times have been averaged over 5 runs.

In our first set of experiments, we have used a synthetic 2-term Lorentz model with the coefficients given in Table I. We have experimented with different levels of $p$-refinement in the discontinuous Galerkin approximation; see Section 2.1. Table II contains the resulting matrix sizes for the standard and direct linearizations along with the computing times for determining the 10 eigenvalues of smallest magnitude for each of 30 different wave vectors distributed along the boundary of the irreducible Brillouin zone. It turns out that our new approach based on direct linearization is faster by a factor between 2.0 and 2.7 .

\begin{tabular}{|c||c|r|}
\hline$\ell$ & 1 & \multicolumn{1}{|c|}{2} \\
\hline$\xi_{\ell}^{2}$ & 98.6960 & 197.3921 \\
\hline$\eta_{\ell}^{2}$ & 55.2698 & 63.1655 \\
\hline$\gamma_{\ell}$ & 0.0063 & 0.1257 \\
\hline \multicolumn{2}{|c|}{$\alpha=2.0$} & \multicolumn{2}{c}{} \\
\cline { 1 - 2 }
\end{tabular}

Table I. Material parameters for the first set of experiments.

\begin{tabular}{|c|c||c|c||c|c|}
\hline \multicolumn{2}{|c||}{ problem size } & \multicolumn{2}{c||}{ standard linearization } & \multicolumn{2}{c|}{ direct linearization } \\
\hline$p$ & \#dofs & size & comp. time (s) & size & comp. time (s) \\
\hline 2 & 288 & 1728 & 15.4 & 720 & 6.4 \\
\hline 4 & 720 & 4320 & 46.1 & 1800 & 16.7 \\
\hline 6 & 1344 & 8064 & 116 & 3360 & 46.7 \\
\hline 8 & 2160 & 12960 & 258 & 5400 & 130 \\
\hline 12 & 4368 & 26208 & 899 & 10920 & 430 \\
\hline 18 & 9120 & 54720 & 3471 & 22800 & 1424 \\
\hline
\end{tabular}

Table II. A comparison of the resulting sizes and computing times between the standard and direct linearizations for a Lorentz model with 2 terms (see Table I). 


\begin{tabular}{|c||r|r|r|r|r|r|r|}
\hline$\ell$ & \multicolumn{1}{|c|}{1} & \multicolumn{1}{c|}{2} & \multicolumn{1}{c|}{3} & \multicolumn{1}{c|}{4} & \multicolumn{1}{c|}{5} & \multicolumn{1}{c|}{6} & \multicolumn{1}{c|}{7} \\
\hline$\xi_{\ell}^{2}$ & 416.6166 & 352.7054 & -339.9124 & 492.5687 & -19.6143 & -527.5597 & 98.0101 \\
\hline$\eta_{\ell}^{2}$ & 92.1086 & 71.6269 & 71.4552 & 227.8301 & 47.4923 & 93.5605 & 121.3762 \\
\hline$\gamma_{\ell}$ & 2.7820 & 0.9597 & 0.9500 & 13.1508 & 9.2697 & 3.2624 & 2.2712 \\
\hline$\alpha=1.143$ & \multicolumn{7}{|l}{} \\
\hline
\end{tabular}

Table III. Material parameters for the second set of experiments, representing $66 \%$ porous silicon, scaled with the lattice constant $a=500 \mathrm{~nm}$.

\begin{tabular}{|c|c||c|c||c|c|}
\hline \multicolumn{2}{|c||}{ problem size } & \multicolumn{2}{c||}{ standard linearization } & \multicolumn{2}{c|}{ direct linearization } \\
\hline$p$ & \#dofs & size & comp. time (s) & size & comp. time (s) \\
\hline 2 & 288 & 4608 & - & 1584 & 14.9 \\
\hline 4 & 720 & 11520 & - & 3960 & 35.2 \\
\hline 6 & 1344 & 21504 & - & 7392 & 89.2 \\
\hline 8 & 2160 & 34560 & - & 11880 & 222 \\
\hline 12 & 4368 & 69888 & - & 24024 & 817 \\
\hline 18 & 9120 & 145920 & - & 50160 & 2081 \\
\hline
\end{tabular}

Table IV. A comparison between the sizes of the standard and direct linearizations for a Lorentz model with 7 terms (see Table III); in the direct case also computing times are given.
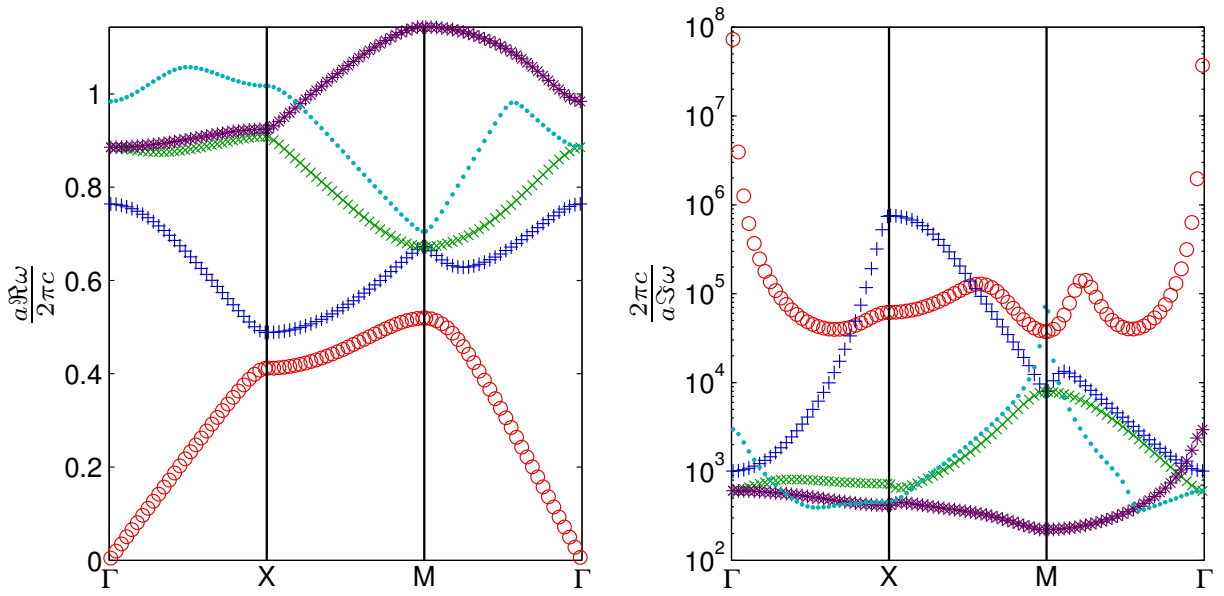

Figure 3. The band diagram computed by our method for the 7 -terms Lorentz model of $66 \%$ porous silicon (see Table III).

In our second set of experiments, we have used 7 Lorentz terms to model the frequencydependent permittivity of $66 \%$ porous silicon [33, Table II, p. 523], scaled to our lattice constant $a=500 \mathrm{~nm}$. The corresponding parameters can be found in Table III. Again, we have computed the 10 eigenvalues of smallest magnitude for each of 30 different wave vectors distributed along the boundary of the irreducible Brillouin zone. Table IV summarizes the results for different levels of $p$-refinement. For the standard linearization, the increased number of Lorentz terms 
does not only severely complicate the analytic derivation of the polynomial coefficients but also results in excessive memory requirements; therefore, we have only stated the expected size of the linearization but no computing times. For the direct linearization, it is interesting to note that the computing times only grow very moderately (roughly by a factor of 2 ) when increasing the number of Lorentz terms from 2 to 7 . This is a consequence of the high level of structure exploitation in the derivation of our new method. Finally, the resulting dispersion relations are depicted in Figure 3.

\section{Conclusions and outlook}

We have proposed an algorithm for solving rational eigenvalue problems arising in band structure calculations for photonic crystals containing frequency-dependent materials. By taking the rich structure of these eigenvalue problems into account, we arrive at linearizations of reduced size, which considerably reduces the computational effort in terms of memory and execution time. Future work includes the efficient handling of non-zero shifts in the linearized eigenvalue solver, which may extend the range of applicability of our method. Moreover, it might be beneficial to combine our method with eigenvalue continuation techniques when repeatedly solving the eigenvalue problem for different but nearby wave vectors, a typical situation in band structure calculations. Unfortunately, the continuation of several eigenvalues for nonlinear eigenvalue problems is presently not very well understood.

\section{Acknowledgements}

We thank the referees for their helpful remarks.

\section{REFERENCES}

1. P. F. Antonietti, A. Buffa, and I. Perugia. Discontinuous Galerkin approximation of the Laplace eigenproblem. Comput. Methods Appl. Mech. Engrg., 195:3483-3503, 2006.

2. D. N. Arnold, F. Brezzi, B. Cockburn, and L. D. Martini. Unified analysis of discontinuous Galerkin methods for elliptic problems. SIAM J. Numer. Anal., 39(5):1749-1779, 2002.

3. W. Axmann and P. Kuchment. An efficient finite element method for computing spectra of photonic and acoustic band-gap materials I. scalar case. J. Comput. Phys., 150:468-481, 1999.

4. T. Betcke and H. Voss. A Jacobi-Davidson type projection method for nonlinear eigenvalue problems. Future generation Computer Systems, 20:363-372, 2004.

5. M. Cessenat. Mathematical Methods in Electromagnetism. Series on Advances in Mathematics for Applied Sciences - Vol. 41. World Scientific Publisher, Singapore, 1996.

6. T. A. Davis. Algorithm 832: UMFPACK v4.3 - an unsymmetric-pattern multifrontal method. ACM Trans. Math. Softw., 30(2):196-199, 2004.

7. D. C. Dobson. An efficient band structure calculations in 2D photonic crystals. J. Comput. Phys., 149:363-376, 1999.

8. C. Engström. On the spectrum of a holomorphic operator-valued function with applications to absorptive photonic crystals. Mathematical Models and Methods in Applied Sciences, 20(8):1319-1341, 2010.

9. C. Engström and Wang M. Complex dispersion relation calculations with the symmetric interior penalty method. International Journal for Numerical Methods in Engineering, 84(7):849-863, 2010.

10. A. Figotin and Y. A. Godin. The computation of spectra of some 2D photonic crystals. J. Comput. Phys., 136:585-598, 1997.

11. A. Figotin and P. Kuchment. Band-gap structure of spectra of periodic dielectric and acoustic media. i scalar model. SIAM J. Appl. Math., 56:68-88, 1996. 
12. I. Gohberg, P. Lancaster, and L. Rodman. Matrix Polynomials. Academic Press, New York, 1982.

13. G. H. Golub and C. F. Van Loan. Matrix Computations. Johns Hopkins Studies in the Mathematical Sciences. Johns Hopkins University Press, Baltimore, MD, USA, third edition, 1996.

14. J. S. Hesthaven and T. Warburton. Nodal Discontinuous Galerkin Methods. Springer-Verlag, Berlin, 2008.

15. K. C Huang, E. Lidorikis, X. Jiang, J. D Joannopoulos, K. A. Nelson, P. Bienstman, and S. Fan. Nature of lossy Bloch states in polaritonic photonic crystals. Phys. Rev. B, 69:195111, 2004.

16. F. Ihlenburg. Finite element analysis of acoustic scattering. Springer-Verlag, Berlin, 1998.

17. J. D. Jackson. Classical Electrodynamics. John Wiley \& Sons, New York, third edition, 1999.

18. J. D. Joannopoulos, S. G. Johnson, J. N. Winn, and R. D. Meade. Photonic Crystals: Molding the Flow of Light. Princeton University Press, Princeton, NJ, USA, second edition, 2008.

19. D. Kressner. A block Newton method for nonlinear eigenvalue problems. Numer. Math., 114(2):355-372, 2009.

20. P. Kuchment. Floquet Theory for Partial Differential Equations. Birkhäuser, Basel, 1993.

21. R. B. Lehoucq, D. C. Sorensen, and C. Yang. ARPACK users' guide. Society for Industrial and Applied Mathematics (SIAM), Philadelphia, PA, 1998.

22. M. Luo, Q. H. Liu, and Z. Li. A spectral element method for band structures of two-dimensional anisotropic photonic crystals. Phys. Rev. E, 79:026705, 2009.

23. D. S. Mackey, N. Mackey, C. Mehl, and V. Mehrmann. Vector spaces of linearizations for matrix polynomials. SIAM J. Matrix Anal. Appl., 28(4):971-1004, 2006.

24. E. Moreno, D. Erni, and Ch. Hafner. Band structure computations of metallic photonic crystals with the multiple multipole method. Phys. Rev. B, 65:155120, 2002

25. F. Odeh and J. B. Keller. Partial differential equations with periodic coefficients and Bloch waves. $J$. Math. Phys., 5:1499-1504, 1964.

26. M. Reed and B. Simon. Methods of modern mathematical physics, volume IV: Analysis of Operators. Academic Press, New York, 1978.

27. K. Sakoda, N. Kawai, T. Ito, A. Chutinan, S. Noda, T. Mitsuyu, and K. Hirao. Photonic bands of metallic systems. I. principle of calculation and accuracy. Phys. Rev. B, 64(4):045116, 2001.

28. E. Saltel and F. Hecht. EMC2 Wysiwyg 2D finite elements mesh generator. In INRIA Report 118, 1995.

29. K. Schmidt and P. Kauf. Computation of the band structure of two-dimensional photonic crystals with hp finite elements. Comput. Methods Appl. Mech. Engrg., 198:1249-1259, 2009.

30. G. L Sleijpen, G. L. Booten, D. R. Fokkema, and H. A. van der Vorst. Jacobi-davidson type methods for generalized eigenproblems and polynomial eigenproblems. BIT 36:3, pages 595-633, 1996.

31. A. Spence and C. Poulton. Photonic band structure calculations using nonlinear eigenvalue techniques. J. Comput. Phys., 204:65-81, 2005.

32. Y. Su and Z. Bai. Solving rational eigenvalue problems via linearization. Technical Report CSE-2008-13, Department of Computer Science, University of California, Davis, 2008.

33. Z. Szabó, G. Kádár, and J. Volk. Band gaps in photonic crystals with dispersion. COMPEL: The International Journal for Computation and Mathematics in Electrical and Electronic Engineering, 24(2):521-533, 2005.

34. K. Vemaganti. Discontinuous Galerkin methods for periodic boundary value problems. Numer Methods Partial Differential Eq., 23(3):587-596, 2007.

35. H. Voss. A maxmin principle for nonlinear eigenvalue problems with application to a rational spectral problem in fluid-solid vibration. Applications of Mathematics, 48:607-622, 2003.

36. H. Voss. A minmax principle for nonlinear eigenproblems depending continuously on the eigenparameter. Numer. Linear Algebra Appl., 16(11-12):899-913, 2009.

37. H. Voss and B. Werner. A minimax principle for nonlinear eigenvalue problems with applications to nonoverdamped systems. Math. Methods Appl. Sci., 4(3):415-424, 1982. 